\title{
Vaginal Delivery after Pelvic Fracture Fixation with a Subcutaneous Anterior Fixator with Review of Literature of Delivery Mode in Pelvic Injuries
}

\author{
Anil Sethi',2, Rahul Vaidya ${ }^{1,2}$, Sarah Schneider ${ }^{1,2}$, Jennifer Fleming1,2, Bryant W. Oliphant ${ }^{1,2}$ \\ ${ }^{1}$ Department of Orthopedics, Detroit Receiving Hospital, Detroit, USA \\ ${ }^{2}$ Wayne State University School of Medicine, Detroit, USA \\ Email: anilsethi09@gmail.com
}

Received 23 July 2014; revised 28 August 2014; accepted 15 September 2014

Copyright @ 2014 by authors and Scientific Research Publishing Inc.

This work is licensed under the Creative Commons Attribution International License (CC BY).

http://creativecommons.org/licenses/by/4.0/

(c) (i) Open Access

\begin{abstract}
Background: The cesarean section rate following a pelvic fracture is more than double the standard norms. This results from residual deformity and a lack of knowledge among obstetricians regarding the ability to deliver vaginally after a pelvic fracture. Aim: To present a case of pelvic fracture that delivered vaginally indicating vaginal delivery is possible even after pelvic fractures that are stabilized surgically with minimal or no residual displacement. Case presentation: We present a patient that delivered vaginally following internal fixation of an unstable pelvic fracture with a retained orthopedic implant that spanned the lower abdomen with a metallic bar. We also discuss the role of cesarean section in patients with pelvic fractures. Conclusion: Women with pelvic fractures may possibly deliver vaginally however decision regarding mode of delivery in such patients should be on a case by case basis.
\end{abstract}

\section{Keywords}

Pelvic Fracture, Infix, Pregnancy

\section{Introduction}

Pelvic fractures in women may result in dyspareunia, dysmenorrhea and affect the ability to deliver a child vaginally [1]. These complaints are more frequent in women with residual pelvic fracture displacement [1] [2]. In a young woman of child bearing age they are commonly the result of high energy trauma. Displaced fractures in 
the present day are treated with surgical stabilization using a variety of devices.

We recently reported a technique to stabilize unstable pelvic fractures with an anterior subcutaneous pelvic fixation (Infix) device [3] [4]. It consists of single screws placed on both side of the pelvis and connected with a subcutaneous rod spanning the lower abdomen. We report on a noncompliant patient treated with this device who delivered vaginally with the device in-situ. The case is of interest since the patient was able to reach term pregnancy and delivered vaginally in the presence of a metallic bar that spanned the lower abdomen. We also discuss the possible effects of pelvic fractures and internal pelvic fixation on delivery.

\section{Case Report}

A 30-year-old G0P0 female involved as a pedestrian in a hit-and-run motor vehicle collision was brought to the emergency department of our hospital by ambulance in November 2007. Radiographs and CT scan of the pelvis demonstrated a fracture of the body of the left sacral ala extending into the L5-S1 facet joint, fracture of the posterior aspect of left ilium extending into a widened sacroiliac joint (Figure 1, Figure 2). The anterior pelvic injury included an ipsilateral inferior and superior pubic ramii fracture. At the time of surgery it was found that the patient had rotational and vertical instability of the left hemi pelvis. The fracture was reduced and a posterior iliosacral screw and an anterior Infix were placed (Figure 3). The patient did well in the immediate postoperative period and was able to ambulate with crutches and weight bearing on the right leg. She was lost to orthopedic follow up for 4 years and was using illicit drugs. During this time the patient had 2 pregnancies that were delivered vaginally. She delivered the first child 321 days following pelvic fixation in a taxi cab and presented soon after delivery with a retained placenta. Maternal pushing assisted with gentle traction resulted in spontaneous delivery of the placenta. There were no vaginal tears or lacerations noted. The second child was also delivered vaginally in another hospital in the city. She had no ante natal care and reported to the hospital at the onset of labor pain. She informed the obstetrician of the Infix device in the lower abdomen who performed an assisted
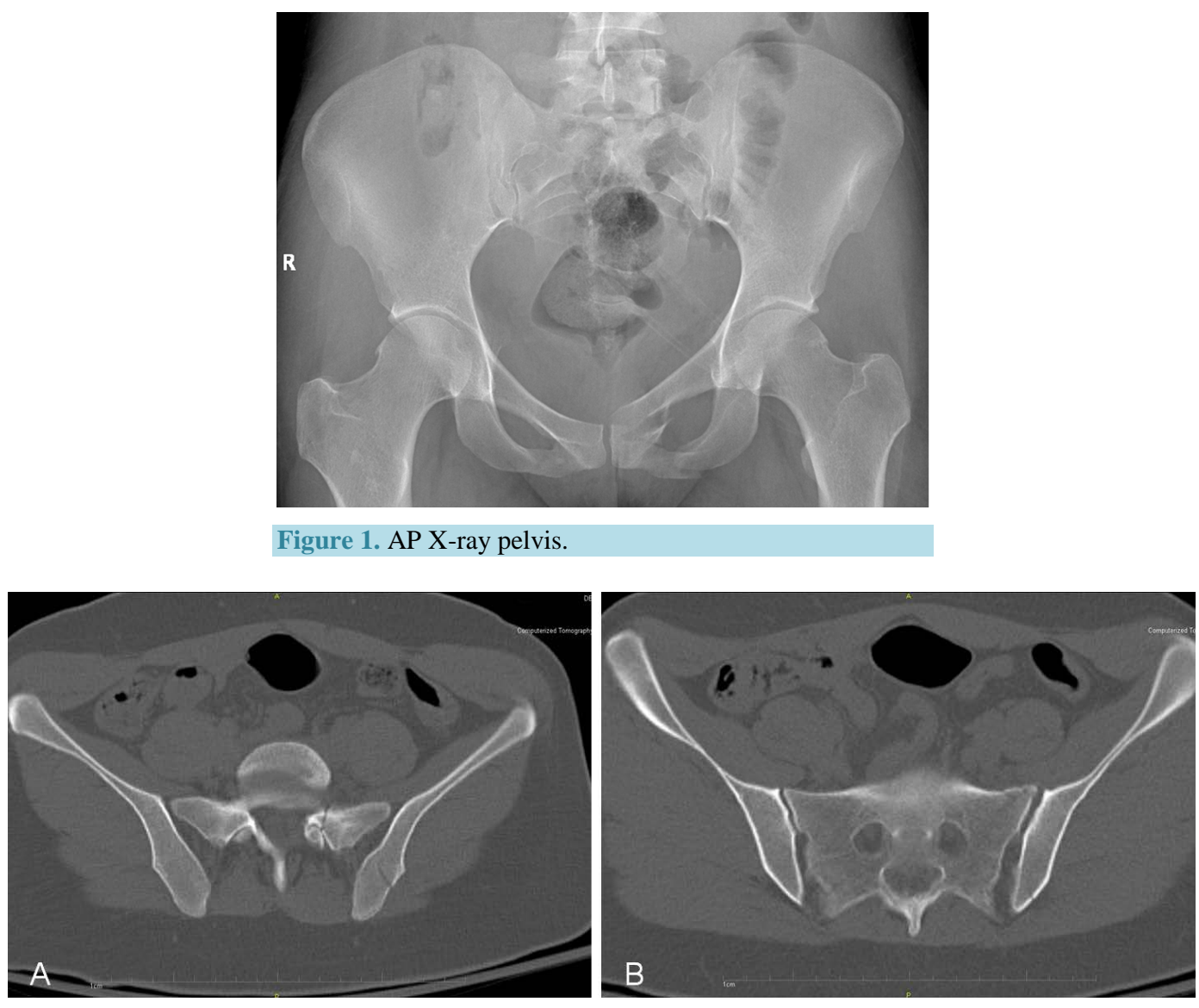

Figure 2. Axial CT scan. 


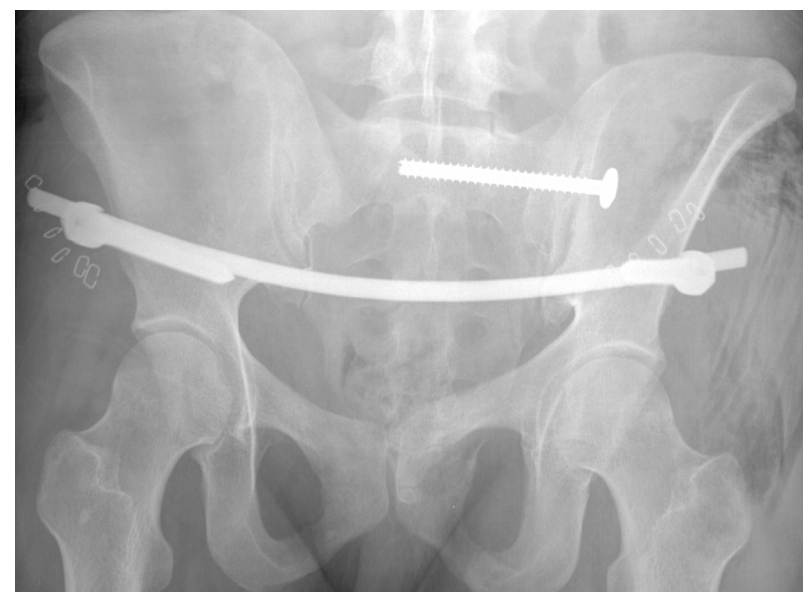

Figure 3. Infix in-situ.

vaginal delivery with vacuum extraction. There were no perinatal complications. She followed up with our service 48 months after the placement of the Infix. Radiographs revealed that the pelvic fractures had healed and the patient was ambulating without any assistive device. The Infix was removed as an outpatient procedure and the patient has been doing well since.

\section{Discussion}

Operative reduction and fixation of unstable pelvic ring injuries yield better anatomical and functional results than non-operative treatment [5] and can be life saving in patients who present with hemodynamic instability from a pelvic injury [6]. Fracture fixation may be achieved with the use of both internal (plates and screws) and external devices. The Infix device stabilizes unstable pelvic fractures with a subcutaneous rod, does not require large incisions as for plates, is more comfortable than an external fixator and has low rates of infection [3] [4]. The device has a bar that spans the lower abdomen and is placed in an area that we have termed the "bikini area" which is formed by the groin creases on the sides and superiorly by a fold of the abdominal tissue [7]. This area is flat in sitting and standing even in obese patients in whom the belly overhangs the superior border of this triangle [3]. It is our premise that in a pregnant patient the gravid uterus also overhangs the bikini area as in an obese individual.

Relaxation of the joints and ligaments occurs during pregnancy around the 10th week of gestation and may persist up to 12 weeks postpartum [8]. This allows the volume of the pelvis to expand during labor and child birth. Though uncommon, an inadequate pelvic volume may result in spontaneous symphyseal rupture or intrapartum separation [9]. After pelvic fracture fixation with posterior and anterior instrumentation the relaxation of the anterior pelvic ligaments at the symphysis pubis or posterior ligaments at the sacroiliac joint may be disturbed. Hence, routine removal of pelvic fixation devices in women of child bearing age has been advocated [2].

Following a pelvic fracture caesarean section rate is more than double the standard norms [8] [9] as there is belief in the obstetric community that women with pelvic fractures cannot deliver vaginally [8]. This has been attributed to several factors including lack of knowledge among obstetricians regarding the ability to deliver vaginally after a pelvic fracture, concern for litigation, and previous results [8]. The caesarean section rate for women in the United States in 2012 was reported at 32.8\% [10]. Previous reports have indicated that women with pelvic fractures have higher rates of cesarean section, urinary tract infections and gynecologic pain in comparison to female multitrauma patients without pelvic fractures [11] [12]. Limb length discrepancy, rotational inequality of the legs and asymmetry of the ischial tuberosities causing difficulty in sitting and ambulation are other complications associated with this injury [11] [12]. Cesarean section is not routinely indicated in patients with a pelvic fracture which is well reduced with or without pelvic implants [2] [9]. However, in displaced pelvic fractures resulting in cephalo-pelvic disproportion there is a need for closer evaluation and some may infact require caesarean section. A recent study reports that patients with initial fracture displacement of $5 \mathrm{~mm}$ or more had an $80 \%$ cesarean section rate compared to $15 \%$ in patients with initial fracture displacement less than $5 \mathrm{~mm}$ [8]. Currently there are no definite guidelines available as to the indications of cesarean section in patients 
with pelvic fracture.

Normally the anterior subcutaneous internal pelvic fixator (INFIX) is removed 3-6 months after the original surgery by which time the pelvic injury has healed. Additionally, due to the subcutaneous placement of the implants the device is extremely easy to remove. It is our premise that this is a useful device when fixing anterior pelvic injuries in women of child bearing age as it is routinely removed after the fracture is healed. This eliminates any retained anterior implants that could potentially tether the anterior structures. Also due to its location and as demonstrated by this non compliant patient, the fixator may be useful when repairing pelvic fractures in women pregnant at the time of injury as it allows the belly to overhang and not interfere with the expanding gravid uterus.

It is our recommendation that all patients be evaluated on a case by case basis since vaginal delivery is possible even after pelvic fractures that are stabilized surgically with minimal or no residual displacement.

\section{Conclusion}

A case of a displaced pelvic fracture stabilized by our new subcutaneous screw and rod technique (Infix) supplemented with posterior fixation that delivered vaginally is presented. Cesarean section after pelvis fractures and pelvic fixation is more likely due to the perception of obstetricians and patients that vaginal delivery may be difficult or contraindicated. There are a few studies that contradict this opinion [1] [9] [10] but no definitive guidelines are available.

\section{References}

[1] Vallier, H.A., Cureton, B.A. and Schubeck, D. (2012) Pelvic Ring Injury Is Associated with Sexual Dysfunction in Women. Journal of Orthopaedic Trauma, 26, 308-313.

[2] Vallier, H.A., Cureton, B.A. and Schubeck, D. (2012) Pregnancy Outcomes after Pelvic Ring Injury. Journal of Orthopaedic Trauma, 26, 302-307.

[3] Vaidya, R., Colen, R., Vigdorchik, J., Tonnos, F. and Sethi, A. (2012) Treatment of Unstable Pelvic Ring Injuries with an Internal Anterior Fixator and Posterior Fixation: Initial Clinical Series. Journal of Orthopaedic Trauma, 26, 1-8. http://dx.doi.org/10.1097/BOT.0b013e318233b8a7

[4] Vaidya, R., Kubiak, E.N., Bergin, P.F., Dombroski, D.G., Critchlow, R.J., Sethi, A. and Starr, A.J. (2012) Complications of Anterior Subcutaneous Internal Fixation for Unstable Pelvis Fractures: A Multicenter Study. Clinical Orthopaedics and Related Research, 470, 2124-2131.

[5] Dunivan, G.C., Hickman, A.M. and Connolly, A. (2009) Severe Separation of the Pubic Symphysis and Prompt Orthopedic Surgical Intervention. Obstetrics Gynecology, 114, 473-475. http://dx.doi.org/10.1097/AOG.0b013e3181998bd1

[6] Najibi, S., Tannast, M., Klenck, R.E. and Matta, J.M. (2010) Internal Fixation of Symphyseal Disruption Resulting from Childbirth. Journal of Orthopaedic Trauma, 24, 732-739. http://dx.doi.org/10.1097/BOT.0b013e3181d70259

[7] Vaidya, R., Oliphant, B., Jain, R., Nasr, K., Siwiec, R., Onwudiwe, N. and Sethi, A. (2013) The Bikini Area and Bikini Line as a Location for Anterior Subcutaneous Pelvic Fixation: An Anatomic and Clinical Investigation. Clinical Anatomy, 26, 392-399. http://dx.doi.org/10.1002/ca.22149

[8] Copeland, C.E., Bosse, M.J., McCarthy, M.L., MacKenzie, E.J., Guzinski, G.M., Hash, C.S. and Burgess, A.R. (1997) Effect of Trauma and Pelvic Fracture on Female Genitourinary, Sexual, and Reproductive Function. Journal of Orthopaedic Trauma, 11, 73-81. http://dx.doi.org/10.1097/00005131-199702000-00001

[9] Cannada, L.K., Pan, P., Casey, B.M., McIntire, D.D., Shafi, S. and Leveno, K.J. (2010) Pregnancy Outcomes after Orthopedic Trauma. The Journal of Trauma, 69, 694-698. http://dx.doi.org/10.1097/TA.0b013e3181e97ed8

[10] http://www.cdc.gov/nchs/data/nvsr/nvsr62/nvsr62_09.pdf\#table21

[11] Papakostidis, C., Kanakaris, N.K., Kontakis, G. and Giannoudis, P.V. (2009) Pelvic Ring Disruptions: Treatment Modalities and Analysis of Outcomes. International Orthopaedics, 33, 329-338. http://dx.doi.org/10.1007/s00264-008-0555-6

[12] Bucholz, R.W., Heckman, J.D., Coutr-Brown, C., Eds. (2006) Rockwood and Green's Fractures in Adults. 6th Edition, Lippincott Williams \& Wilkins, Philadelphia. 
Scientific Research Publishing (SCIRP) is one of the largest Open Access journal publishers. It is currently publishing more than 200 open access, online, peer-reviewed journals covering a wide range of academic disciplines. SCIRP serves the worldwide academic communities and contributes to the progress and application of science with its publication.

Other selected journals from SCIRP are listed as below. Submit your manuscript to us via either submit@scirp.org or Online Submission Portal.
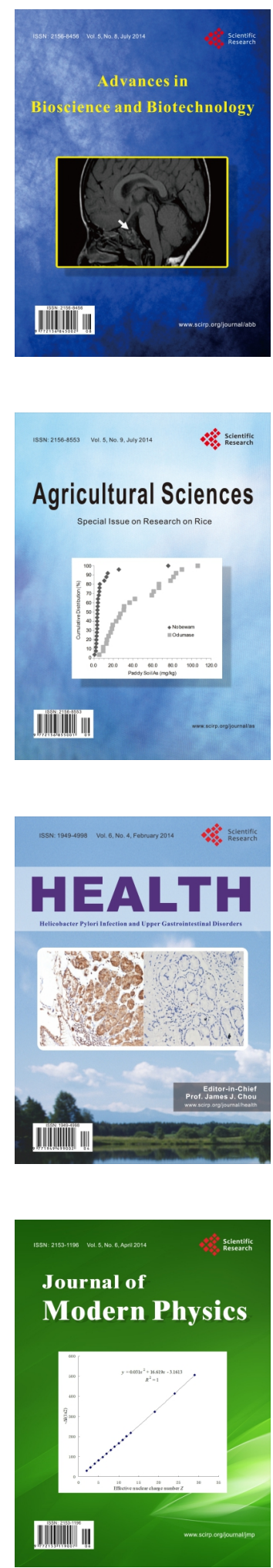
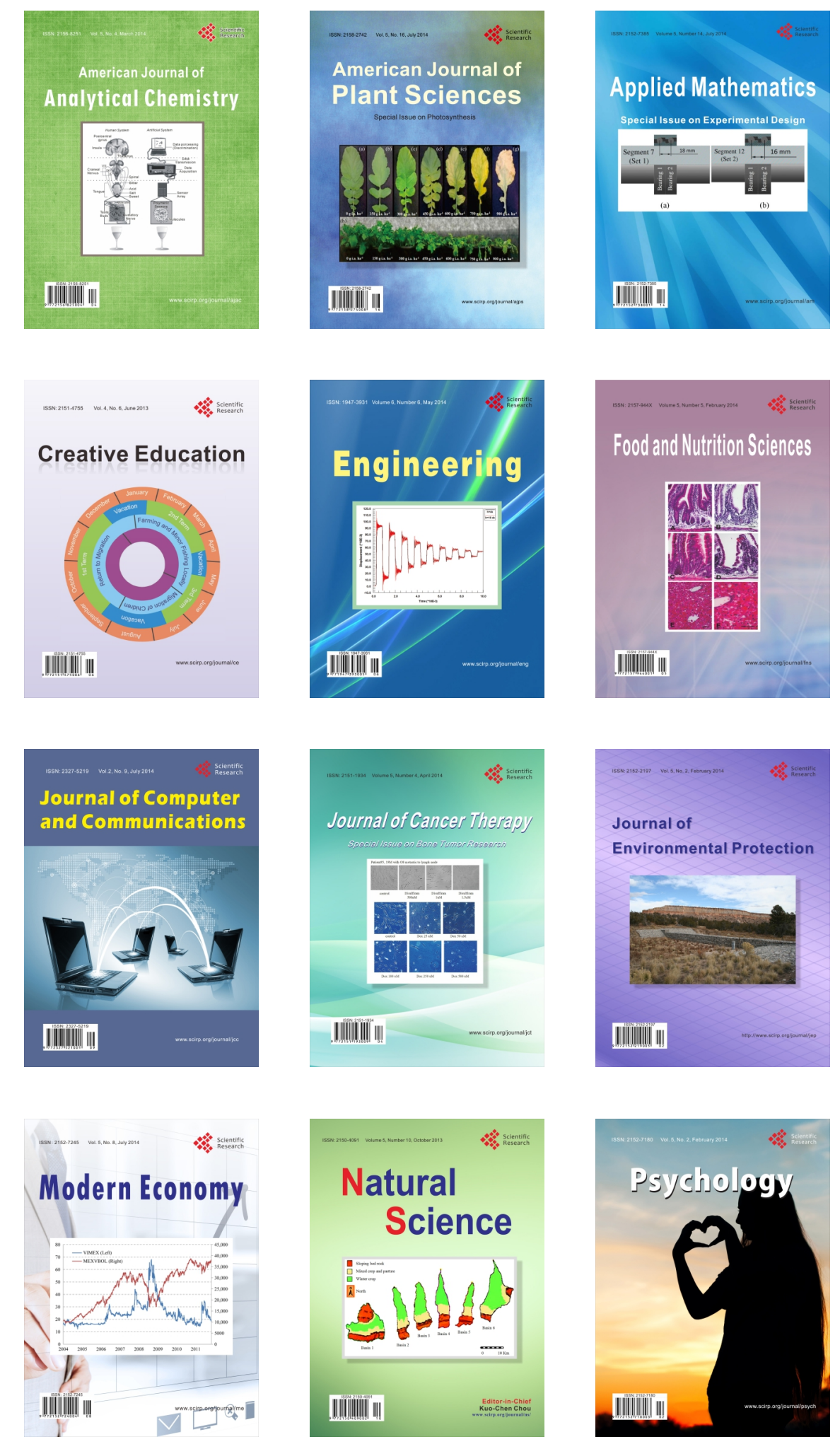